\title{
Lungenembolie (LE)
}

\section{Definition und klinische Problematik}

Bei der Lungenembolie (LE) handelt es sich um eine partielle oder vollständige Verlegung der Lungenarterien durch eingeschwemmte Blutgerinnsel aus der peripheren venösen Strombahn.

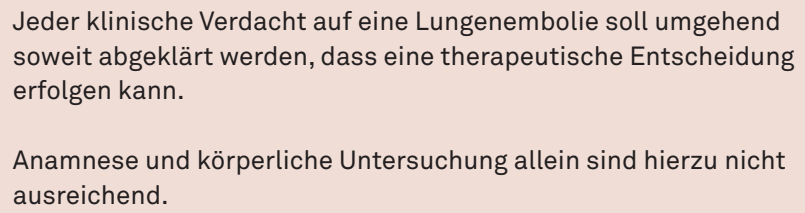

Anamnese und körperliche Untersuchung allein sind hierzu nicht ausreichend.

Bei Verdacht auf Lungenembolie soll eine initiale Risikostratifizierung erfolgen, um zwischen hämodynamisch stabilen und instabilen Patienten zu unterscheiden.

In Europa sind schätzungsweise bis zu 370.000 Todesfälle jährlich mit einer akuten LE assoziiert [57]. Die Frühletalität ist abhängig vom Ausmaß der LE, dem Vorliegen und Schweregrad einer rechtsventrikulären (RV)

Tabelle IV: Validierter klinischer Score zur Ermittlung der klinischen Wahrscheinlichkeit einer Lungenembolie: Wells-Score [306]

\begin{tabular}{lcc}
\hline \multicolumn{2}{c}{ Wells-Score } & \multicolumn{2}{c}{ Punkte } \\
\hline \multirow{2}{*}{ Kriterien } & $\begin{array}{c}\text { Original- } \\
\text { version }\end{array}$ & $\begin{array}{c}\text { Vereinfachte } \\
\text { Version }\end{array}$ \\
\hline Frühere TVT oder LE & 1,5 & 1 \\
Frische Operation oder & 1,5 & 1 \\
Immobilisation & 1 & 1 \\
Tumorerkrankung & 1 & 1 \\
Hämoptyse & 1,5 & 1 \\
Herzfrequenz $\geq 100$ Schläge & & 1 \\
pro Minute & 3 & 1 \\
Klinische Zeichen einer TVT & 3 & 1 \\
Alternative Diagnose \\
unwahrscheinlicher als LE
\end{tabular}

Klinische Wahrscheinlichkeit

\begin{tabular}{lcc}
\hline 3 Ebenen-Score & \\
\hline Niedrig & $0-1$ & - \\
Mittel/intermediär & $2-6$ & - \\
Hoch & $\geq 7$ & - \\
\hline
\end{tabular}

\begin{tabular}{lcc}
\hline Dichotomisierter Score & & \\
\hline LE unwahrscheinlich & $0-4$ & $0-1$ \\
LE wahrscheinlich & $\geq 5$ & $\geq 2$ \\
\hline
\end{tabular}

TVT = Venenthrombose, LE = Lungenembolie
Dysfunktion und eventuellen kardiopulmonalen Begleiterkrankungen (Komorbidität); bis zu 90 \% aller Todesfälle ereignen sich akut innerhalb von 2 Stunden nach Symptombeginn $[235,273]$. Bei den Überlebenden ist die Prognose insbesondere bei Auftreten von Rezidivembolien und Rechtsherzversagen ungünstig [101, 137]. Durch eine adäquate Antikoagulation lässt sich die LE-bedingte Letalitätsrate in den ersten 3 Monaten auf $<2 \%$ senken [160]. Daraus ergibt sich die Notwendigkeit einer unverzüglichen und konsequent durchgeführten Diagnostik bei klinischem Verdacht auf eine akute LE. Bei begründetem Verdacht kann es notwendig werden, eine Therapie bereits einzuleiten, bevor die Diagnose gesichert ist.

In den folgenden Abschnitten zur Diagnostik und Therapie der Lungenembolie orientiert sich diese Leitlinie an den ESC Guidelines on Pulmonary Embolism aus dem Jahr 2014 [151].

\section{Diagnostik der Lungenembolie}

\section{Klinische Diagnostik}

Die häufigsten klinischen Symptome einer akuten Lungenembolie (LE) sind Dyspnoe mit plötzlichem Beginn, Brustschmerz, Synkope oder Präsynkope und Hämoptyse [196, 223, 296, 306]. Der physikalische Befund an der Lunge ist typischerweise unauffällig.

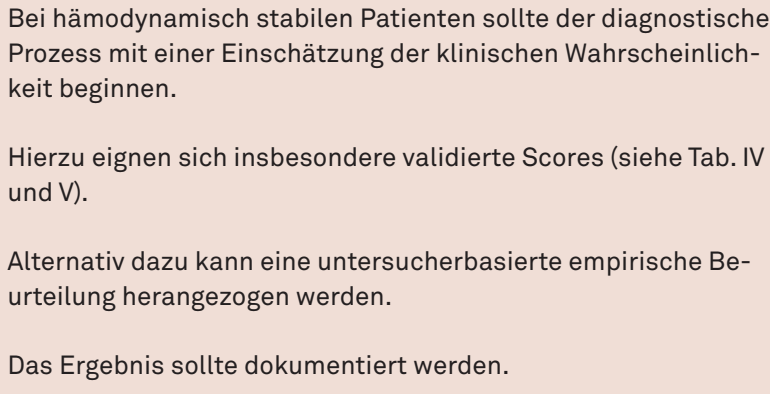

Zur Basisdiagnostik zählen die Vitalparameter, eine Röntgen-Thoraxübersicht in 2 Ebenen, ein EKG und die Blutgasanalyse. Jeder Parameter für sich betrachtet bleibt unzureichend [196]. Alle zusammen sind aber geeignet, einen Gesamteindruck von der Situation zu vermitteln.

In den Begriff ,klinische (oder Prätest-)Wahrscheinlichkeit" gehören Elemente der Anamnese und des körperlichen Untersuchungsbefundes sowie der Basisdiagnostik. Die ausdrückliche Dokumentation der klinischen Wahrscheinlichkeit ist ein wichtiger erster Schritt, da hierdurch das weitere Vorgehen entscheidend geprägt wird. Am besten untersucht und validiert sind der sog. Wells-Score sowie der revidierte Genfer Score [166, 306]; (Tab. IV und V). Beide Scores wurden nach ihrer Erstbeschreibung überabeitet und stehen auch in vereinfachten Versionen 
zur Verfügung [99, 147]; (Tab. IV und V). Die Aussagefähigkeit der Scores und ihrer vereinfachten Versionen ist vergleichbar: Etwa 10\% aller Patienten mit errechneter niedriger klinischer Wahrscheinlichkeit, 30\% der Patienten mit mittlerer/intermediärer klinischer Wahrscheinlichkeit und $65 \%$ jener mit hoher klinischer Wahrscheinlichkeit werden am Ende des diagnostischen Prozesses eine (bestätigte) LE haben [47]. Wenn eine Dichotomisierung der Scores (=LE wahrscheinlich versus $L E$ unwahrscheinlich) verwendet wird, beträgt die Inzidenz einer bestätigten Lungenembolie in der Gruppe LE unwahrscheinlich 12\% [47].

\section{D-Dimer-Tests}

Die Testverfahren weisen eine unterschiedliche Treffsicherheit auf. Die Sensitivität reicht bei einzelnen Tests bis über 95\%; die Spezifität ist jedoch relativ gering. Wie bei der Venenthrombose (TVT) ist ein positiver D-Dimer-Befund unspezifisch. Dagegen hat ein negativer D-DimerTest einen hohen Erkenntniswert für den Ausschluss einer Lungenembolie (LE); er ist in seiner prädiktiven Bewertung sogar höher einzuschätzen als bei der TVT.

Bei hämodynamisch stabilen Patienten soll ein D-Dimer-Test nur nach vorheriger Einschätzung der klinischen Wahrscheinlichkeit durchgeführt werden.

Bei nicht hoher (niedriger/ mittlerer) klinischer Wahrscheinlichkeit und normalen D-Dimeren ist keine weitere LungenembolieDiagnostik erforderlich.

Bei hoher klinischer Wahrscheinlichkeit soll kein D-Dimer-Test durchgeführt werden.

In einer klinischen Management-Studie wurde ein quantitativer ELISA mit einem Grenzwert (Cut off-value) von $500 \mu \mathrm{g} / \mathrm{l}$ als alleiniger erster diagnostischer Schritt bei ambulanten Patienten mit Verdacht auf LE eingesetzt [214]. Bei den nicht therapierten Patienten mit negativem Ergebnis betrug die Rate an Thromboembolien nach 3 Monaten 0,9\% (95\% Konfidenzintervall 0,2\%-2,7\%). In anderen Studien wurde von vornherein die Kombination aus D-Dimer-Test und klinischer Wahrscheinlichkeit eingebunden. Das bedeutet eine zusätzliche Absicherung und wird allgemein für erforderlich gehalten. Bei Patienten mit niedriger klinischer Wahrscheinlichkeit erwies sich ein einfacher qualitativer Bedside-Test zur D-DimerBestimmung in Kombination mit der klinischen Wahrscheinlichkeit gegenüber dem aufwändigeren quantitativen ELISA-Test in seiner Aussagekraft als vergleichbar [274].

Die Verwendung eines altersadaptierten Grenzwerts (Alter $\times 10 \mu \mathrm{g} / \mathrm{l}$ bei einem Lebensalter $>50$ Jahre) kann ohne signifikanten Verlust der Sensitivität und des negativ-prädiktiven Wertes die diagnostische Spezifität erhöhen [253]. Dieses Konzept wurde kürzlich in einer prospektiven Studie an 3.346 Patienten erfolgreich getestet: Die Verwendung des altersadaptierten D-Dimer-Grenzwertes hat - im Vergleich zum Standardwert von $500 \mu \mathrm{g} / \mathrm{l}$ - den Anteil der Patienten, bei denen eine LE ohne weiterführende Diagnostik ausgeschlossen werden konnte, von
Tabelle V: Validierter Score zur Ermittlung der klinischen Wahrscheinlichkeit einer Lungenembolie: Genfer Score [167]

\begin{tabular}{|c|c|c|}
\hline \multicolumn{3}{|c|}{ Revidierter Genfer Score } \\
\hline \multirow[b]{2}{*}{ Kriterien } & \multicolumn{2}{|c|}{ Punkte } \\
\hline & $\begin{array}{l}\text { Original- } \\
\text { version }\end{array}$ & $\begin{array}{l}\text { Vereinfachte } \\
\text { Version }\end{array}$ \\
\hline Alter $>65$ Jahre & 1 & 1 \\
\hline Frühere TVT oder LE & 3 & 1 \\
\hline $\begin{array}{l}\text { Operation oder Knochenfraktur } \\
\text { innerhalb des letzten Monats }\end{array}$ & 2 & 1 \\
\hline Aktive Tumorerkrankung & 2 & 1 \\
\hline Einseitiger Beinschmerz & 3 & 1 \\
\hline Hämoptyse & 2 & 1 \\
\hline \multicolumn{3}{|l|}{ Herzfrequenz: } \\
\hline 75-94 Schläge pro Minute & 3 & 1 \\
\hline z95 Schläge pro Minute & 5 & 2 \\
\hline $\begin{array}{l}\text { Schmerz bei Palpation entlang } \\
\text { einer tiefen Beinvene, einseitiges } \\
\text { Ödem }\end{array}$ & 4 & 1 \\
\hline \multicolumn{3}{|c|}{ Klinische Wahrscheinlichkeit } \\
\hline \multicolumn{3}{|l|}{3 Ebenen-Score } \\
\hline Niedrig & $0-3$ & $0-1$ \\
\hline Mittel / intermediär & $4-10$ & $2-4$ \\
\hline Hoch & $\geq 11$ & $\geq 5$ \\
\hline \multicolumn{3}{|l|}{ Dichotomisierter Score } \\
\hline LE unwahrscheinlich & $0-5$ & $0-2$ \\
\hline LE wahrscheinlich & $\geq 6$ & $\geq 3$ \\
\hline
\end{tabular}

TVT = Venenthrombose; LE = Lungenembolie

$6,4 \%$ auf $30 \%$ erhöht; der Prozentsatz falsch-negativer Befunde hat dabei nicht zugenommen [242].

Auf keinen Fall soll bei Patienten mit hoher klinischer Wahrscheinlichkeit zusätzlich ein D-Dimer-Test eingesetzt werden. Da sein negativ-prädiktiver Wert in dieser Situation nicht ausreicht, ist in jedem Fall eine Bildgebung erforderlich.

\section{Computertomographie}

Die computertomographische Pulmonalisangiographie (CTPA) ist universell verfügbar und liefert rasch einen Befund. Die Sensitivität gegenüber der konventionellen Pulmonalisangiographie liegt bei 94 bis $96 \%$ [251].

Wenn eine Bildgebung erforderlich ist, soll eine

CT-Pulmonalisangiographie durchgeführt werden.

Die Methode wird in Mehrschicht-Spiraltechnik (MSCT) eingesetzt; hierbei sollten mindestens 4 Schichten per Umlauf akquiriert werden. Mit der MSCT konnte die Sensitivität für den Nachweis peripherer Embolien deutlich 
erhöht werden [97, 233, 251]. Die Möglichkeit, einen Datensatz mit einer Auflösung im Submillimeterbereich zu generieren, ist mit Geräten gegeben, die 16 Schichten (sog. , 16-Zeiler") oder mehr pro Umlauf generieren [312]. Die Einzelschicht-Spiraltechnik ist nicht mehr zeitgemäß.

Der im Vergleich zur Szintigraphie höheren Strahlenbelastung (s. Tab. VIII) bei der CTPA kann durch Maßnahmen zur Dosisreduktion (Röhrenstrommodulation, individualisierte Protokolle, niedrige Röhrenspannung) begegnet werden $[254,280,312]$. Weitere Techniken wie die iterative Rekonstruktion sowie eine ultraschnelle Datenakquisition führen zu einer weiteren Reduktion der Strahlendosis.

In einer 2005 veröffentlichten prospektiven Studie wurde ermittelt, dass der Ausschluss einer Lungenembolie (LE) auf dem Boden der klinischen Wahrscheinlichkeit, des D-Dimer-Tests und der CTPA ohne weitere bildgebende Verfahren ein 3-Monats-Rezidivrisiko von nur $1,5 \%$ aufweist [216]. In Übereinstimmung mit diesem Ergebnis lieferte eine prospektive Management-Studie an 3.306 Patienten mit klinisch vermuteter LE eine solide Basis für einen vereinfachten CTPA-gestützten diagnostischen LE-Algorithmus [288]. Mit Hilfe des dichotomisierten Wells-Scores (=LE wahrscheinlich bzw. unwahrscheinlich bei einem Score von 4 als Grenzwert; s. Tab. IV) und eines D-Dimer-Tests (ELISA) konnte eine LE bei $32 \%$ dieser Patienten ohne weitere Diagnostik ausgeschlossen werden. Die übrigen Patienten wurden einer CTPA mit Mehrschicht-Spiraltechnik unterzogen. Die Patienten mit Ausschluss einer LE in der CTPA erhielten bis auf sehr wenige Ausnahmen keine Antikoagulation; in dieser Gruppe betrugen die 3-Monats-Rate von VTERezidiven $1,3 \%$ und die LE-assoziierte 3-Monats-Mortalitätsrate $0,5 \%$. Insgesamt erlaubte diese diagnostische Strategie eine therapeutische Entscheidung in $98 \%$ der Fälle.

Eine routinemäßige Kombination der CTPA mit einer indirekten CT-Phlebographie in einer Sitzung ist nicht zu empfehlen, da diese mit einer vermehrten Strahlenbelastung ohne signifikante Verbesserung der diagnostischen Spezifität oder des negativ-prädiktiven Wertes der CTPA einhergeht [106, 126, 135, 275].

Für die CTPA sind auch Scores beschrieben [183, 232], die eine Einschätzung des Schweregrades der LE erlauben. Eine direkte, verlässliche Methode ist das Verhältnis der enddiastolischen Durchmesser des rechten gegenüber dem linken Ventrikel (RV/LV): ist dieses Verhältnis 1:1 oder grösser, besteht computertomographisch - in Analogie zur Echokardiographie - ein eindeutiger Hinweis auf das Vorliegen einer rechtsventrikulären Belastung [189].

Die hohe diagnostische Sensitivität der CTPA bedingt auch, dass zunehmend subsegmentale Emboli bei asymptomatischen Patienten entdeckt werden, deren klinische und therapeutische Relevanz unklar ist [310].

\section{Szintigraphie}

Die Ventilations-/Perfusionsszintigraphie ist ein etabliertes Verfahren zum Ausschluss oder Nachweis einer Lungenembolie (LE). Bei technisch zeitgemäßer Durchführung weist sie eine hohe Sensitivität von $92 \%$ und eine Spezifität von $91 \%$ auf [169].

Die kombinierte Ventilations-/Perfusionsszintigraphie kann beim hämodynamisch stabilen Patienten als Alternative zur CT-Pulmonalisangiographie eingesetzt werden.

Diese sollte als Teil eines diagnostischen Algorithmus verwendet werden, wenn die CT-Pulmonalisangiographie bei individueller Abschätzung zu risikoreich erscheint.

Die Ventilationsuntersuchung erfolgt in der Regel nach Inhalation von Tc-99m-markierten Aerosolen oder Tc-99mmarkierten Nanopartikeln (Technegas), sehr selten mit Edelgasen (Xe-133). Die Perfusionsuntersuchung wird nach intravenöser Gabe von mit Tc-99m-markiertem makroaggregierten Albumin (MAA) durchgeführt, welches einen kleinen, aber repräsentativen Anteil der Lungenstrombahn markiert und damit die Lungenperfusion abbildet. Allergische Reaktionen auf Tc-99m-MAA sind äußerst selten. Eine Niereninsuffizienz stellt - unabhängig vom Schweregrad keine Kontraindikation für die Tc-99m-MAA Gabe dar. Legt man ein gebräuchliches Untersuchungsprotokoll zu Grunde, so ist die Strahlenexposition der kombinierten Ventilations-/ Perfusionsszintigraphie mit etwa 1,8 mSv gering.

Eine unauffällige Perfusionsstudie schließt eine Lungenembolie mit hoher Wahrscheinlichkeit aus [268]. Anhand der Ventilationsstudie werden Perfusionsdefizite als Mismatch-Befunde (=erhaltene Ventilation bei reduzierter Perfusion) oder als Match-Befunde (=gleichsinnig reduzierte Ventilation und Perfusion) unterschieden. Mismatch-Befunde sprechen für eine Lungenembolie und Matchbefunde dagegen. Bei nicht durchgeführter Ventilationsuntersuchung können Perfusionsauffälligkeiten trotz vorliegendem Röntgen-Thorax-Befund - oft nicht abschließend beurteilt werden, was zu einem höheren Anteil diagnostisch nicht verwertbarer Befunde führt.

Die Ventilations-/Perfusionsszintigraphie sollte in 3DSPECT-Technik erfolgen, da so - gegenüber der planaren Bildgebung - auch kleinere Befunde überlagerungsfrei abgebildet werden können [112, 234]. Die Befundbeschreibung sollte ganz konkret den Nachweis oder Ausschluss eines embolietypischen Befundes wiedergeben; die Angabe einer „wahrscheinlich vorliegenden“ Lungenembolie ist unzureichend. Es konnte kürzlich gezeigt werden, dass bei Einsatz definierter Kriterien zum Embolienachweis $(\geq 1$ segmentaler oder 2 subsegmentale Mismatch-Befunde) mit der Ventilations-/Perfusions-SPECT eine Sensitivität von $92 \%$ und eine Spezifität von 91\% gegenüber einem klinischen Follow up mit Einschluss der CT-Pulmonalisangiographie erreicht werden können [169].

Ein neuer diagnostischer Ansatz ist die Kombination der Ventilations-/Perfusionsszintigraphie mit der low-dose-CT an einem SPECT-CT Hybridgerät [112]. Dabei blieb die bekanntermaßen hohe Sensitivität von $97 \%$ erhalten bei gleichzeitigem Anstieg der Spezifität auf 100\%, da falsch positive Befunde nicht embolischer Genese auf Grund struktureller Auffälligkeiten eliminiert werden konnten. Hier bleiben größere Studien abzuwarten, um 
den Zugewinn durch die Kombination beider Methoden zu validieren.

\section{Magnetresonanztomographie / MR-Angiographie}

Magnetresonanztomographie (mit speziellen MR-Perfusionssequenzen) und MR-Angiographie gelten als innovative Untersuchungsverfahren bei Verdacht auf Lungenembolie. Ihre Aussagekraft ist wegen der eingeschränkten Studienlage bei begrenzter Verfügbarkeit des Verfahrens derzeit noch nicht abschließend beurteilbar [32].

Bei entsprechender Expertise stellt die MR-Angiographie eine interessante Alternative zu den etablierten Untersuchungsverfahren dar und kann in Einzelfällen (z.B. schwere allergische Reaktion auf jodhaltige Kontrastmittel, Schwangerschaft) erwogen werden. Der Einsatz bleibt vorerst spezialisierten Zentren vorbehalten, die aufgrund ihrer Logistik und/oder ihres Patientenkollektivs diese Methode bereits breit einsetzen [27, 28, 311].

\section{Pulmonalisangiographie}

Die selektive Pulmonalisangiographie gilt als der historische Goldstandard in der Diagnostik der Lungenembolie (LE). Bei korrekter Durchführung wird davon ausgegangen, dass ein positives Angiogramm die LE beweist und ein negatives Angiogramm diese ausschließt [38]. Nach Platzierung der Katheterspitze im Truncus pulmonalis wird bei der digitalen Angiographie in Subtraktionstechnik (DSA) das Kontrastmittel direkt in die Lungenarterien injiziert.

Durch die neuen bildgebenden Verfahren, insbesondere die CTPA, hat die Pumonalisangiographie wesentlich an Bedeutung verloren und ist nur noch in seltenen Fällen indiziert, beispielsweise bei instabilen Patienten mit absoluter Kontraindikation gegen eine Thrombolyse, bei denen eine notfallmäßige kathetertechnische Thrombusaspiration oder-fragmentation geplant ist.

\section{Sonographie der Beinvenen}

Die hohe Verfügbarkeit des Kompressionsultraschalls mit standardisierter Darstellung des gesamten Venensystems beider Beine erlaubt es, das Verfahren auch zur Abklärung eines Verdachts auf Lungenembolie (LE) einzusetzen, zumal es sich um eine nicht-invasive Methode handelt. Bei Patienten mit hoher klinischer Wahrscheinlichkeit auf eine LE bzw. bei Patienten mit nicht-hoher klinischer Wahrscheinlichkeit, jedoch positivem D-Dimer-Test kann der Nachweis einer Beinvenenthrombose als Bestätigung einer vermuteten LE gelten. Dies trifft sowohl auf symptomatische als auch auf asymptomatische Beinvenenthrombosen zu.

Die Sonographie der Beinvenen kann beim hämodynamisch stabilen Patienten zur Abklärung des Verdachts auf Lungenembolie eingesetzt werden.

Bei Nachweis einer Beinvenenthrombose kann der Verdacht auf eine Lungenembolie als bestätigt angesehen werden.

Bei nachgewiesener Thrombose ist die Indikation zu einer therapeutischen Antikoagulation gegeben. Bezüglich
Art und Dauer der Behandlung ergeben sich keine Unterschiede bei alleiniger Thrombose gegenüber alleiniger Lungenembolie bzw. der Kombination aus beiden Krankheitsentitäten, solange Kreislaufstabilität besteht (s. „Therapie der Lungenembolie").

Die Sonographie der Beinvenen ist daher ein sinnvoller diagnostischer Schritt für alle hämodynamisch stabilen Patienten, bei denen eine Bildgebung bezüglich des Verdachts auf eine Lungenembolie erforderlich ist. Das trifft insbesondere dann zu, wenn z.B. aus strahlenhygienischen Gründen oder wegen einer eingeschränkten Nierenfunktion auf die speziellen bildgebenden Verfahren der Lungenstrombahn (CTPA, Szintigraphie) möglichst verzichtet werden soll.

\section{Echokardiographie}

Wenn bei einem hämodynamisch instabilen Patienten der Verdacht auf eine akute Lungenembolie (LE) besteht, ermöglicht die transthorakale Echokardiographie zunächst den Nachweis bzw. den Ausschluss differentialdiagnostisch wichtiger kardialer Krankheitsbilder, insbesondere eines linksventrikulären systolischen und/oder diastolischen Pumpversagens, eines Klappenvitiums oder eines tamponierenden Perikardergusses. Darüber hinaus besteht die Rolle der Echokardiographie im Nachweis der RVDruckbelastung und -Dysfunktion, welche die Ursache der hämodynamischen Instabilität (kardiogener Schock) und damit einen entscheidenden Faktor für einen ungünstigen klinischen Verlauf der LE in der Akutphase darstellt [178]. Andererseits kann bei normalem echokardiographischem Befund eine hämodynamisch wirksame, den Patienten vital bedrohende LE mit großer Sicherheit ausgeschlossen werden $[109,138]$.

Bei hämodynamisch instabilen Patienten mit Verdacht auf Lungenembolie sollte die Echokardiographie frühzeitig zur Diagnostik durchgeführt werden, wenn eine CT-Pulmonalisangiographie nicht sofort durchführbar ist.

Auch bei einem hämodynamisch stabilen Patienten sollte die Größenrelation von rechtem zu linkem Ventrikel (RV/ LV Ratio) bestimmt werden, um das Akutrisiko des Patienten auf der Basis einer rechtsventrikulären Dysfunktion weiter zu stratifizieren und somit eine Grundlage für die Überwachungsintensität und für weitere Therapieentscheidungen zu schaffen. Das ist mit der CTPA im Rahmen der LE-Diagnostik oder mit der transthorakalen Echokardiographie im Anschluss an die LE-Diagnostik möglich.

Die rechtsventrikuläre Dysfunktion ermöglicht eine prognostische Einschätzung des Krankheitsverlaufs [61 295, 297]. Sie wird echokardiographisch in unterschiedlicher Weise definiert. Dafür werden die nachfolgenden Kriterien einzeln oder in Kombination herangezogen: eingeschränkte Wandbewegung des rechten Ventrikels, RVDilatation, abnormale (paradoxe) Bewegung des interventrikulären Septums während des Herzzyklus, reduzierte systolische Bewegung des Trikuspidalklappenrings (TAPSE), Nachweis einer Trikuspidalklappeninsuffizienz mit darüber abgeschätztem erhöhten systolischen pulmonalar- 
teriellen Druck, sowie Erweiterung (durch Stauung) der Vena cava inferior [35, 156, 231]. Die Spezifität der echokardiographischen Parameter ist allerdings unter bestimmten klinischen Bedingungen reduziert. Das trifft zu auf Patienten mit vorbestehender pulmonaler Hypertonie, die präkapillär (wie z.B. bei chronischer Lungenerkrankung oder chronisch-thromboembolischer pulmonaler Hypertension) oder postkapillär (wie z.B. bei linksventrikulärem Pumpversagen oder einem signifikanten Mitralklappenvitium) verursacht wird [248]. Ferner können ausgeprägtes Übergewicht, Lungenemphysem oder maschinelle Beatmung die Aussagefähigkeit der Methode einschränken.

Die transösophageale Echokardiographie bietet gegenüber der transthorakalen Beschallung eine bessere örtliche Auflösung. Sie kommt somit für maschinell beatmete Patienten sowie für Patienten mit unzureichender Schallbarkeit (ausgeprägtes Übergewicht, Lungenemphysem) in Frage. Neben der rechtsventrikulären Dysfunktion können mit der transösophagealen Echokardiographie intrakardiale und zentrale pulmonalarterielle Emboli mit hoher Sensitivität und Spezifität nachgewiesen werden [293].

\section{Sonographie der Lungen}

Der Lungenultraschall (LUS) kann eine Lungenembolie (LE) nachweisen [186, 294]. Führendes sonographisches Kriterium ist die Darstellung von mindestens zwei dreieckigen oder runden Läsionen, subpleural im Lungenparenchym gelegen. Die Mehrzahl der Herde ist in den dorsobasalen Lungenabschnitten zu finden [185]. Zur differentialdiagnostischen Abgrenzung kleiner pleuropneumonischer Herde kann die Kontrastmittelsonographie hilfreich sein [107].

Der Lungenultraschall kann bei hämodynamisch stabilen Patienten bei der Abklärung des Verdachts auf Lungenembolie eingesetzt werden.

Der Lungenultraschall kann jedoch eine Lungenembolie nicht ausschließen. Bei der Kombination von LUS mit der Kompressionssonographie der Beinvenen und der Echokardiographie lässt sich für den Nachweis einer thromboembolischen Krankheit eine Sensitivität von $90 \%$ bei einer Spezifität von $86 \%$ erzielen $[184,200]$. Diese nichtinvasive Untersuchungskombination kann von Vorteil sein, um in besonders vulnerablen Patientengruppen (Kinder, Schwangere) bildgebende Verfahren mit Strahlenund Kontrastmittelbelastung einzusparen und sie kann bei Kontraindikationen zur CTPA wie schwerer Niereninsuffizienz, Hyperthyreose und Kontrastmittelallergie als Alternative erwogen werden. Falls eine ausreichende Expertise vorliegt gilt dies besonders in der präklinischen Situation (Hausarzt, Notarzt), in der Notaufnahme und auf der Intensivstation, wenn die CTPA nicht unmittelbar verfügbar ist [200].

Bei fehlendem Nachweis einer LE im Lungenultraschall sind unbedingt weitere bildgebende diagnostische Verfahren (CTPA, Szintigraphie) erforderlich. Auf keinen Fall darf ein Patient allein auf der Basis eines negativen LUSBefundes ohne Antikoagulation belassen werden.

\section{Diagnostische Algorithmen}

Der konsequente Einsatz standardisierter diagnostischer Algorithmen senkt sowohl die akute Letalität als auch die 3-Monats-Rezidivrate einer Lungenembolie (LE) [244]. Die Wahl eines diagnostischen Algorithmus soll sich dabei nicht nur nach der Verfügbarkeit der einzelnen Untersuchungen vor Ort richten, sondern insbesondere auch nach dem hämodynamischen Status des Patienten und somit dem klinischen Schweregrad der vermuteten LE.

Die vorgenannten Methoden (klinische Wahrscheinlichkeit, D-Dimer-Test, CT-Pulmonalisangiographie, Szintigraphie, Ultraschallverfahren) sollen in Abhängigkeit vom hämodynamischen Status des Patienten und der damit verbundenen Dringlichkeit zu einem Algorithmus verbunden werden.

Eine Empfehlung für den hämodynamisch instabilen Patienten mit persistierender arterieller Hypotension oder Schock (hohes klinisches Risiko) gibt Abbildung 2.

Eine Empfehlung für den hämodynamisch stabilen, normotensiven Patienten (nicht hohes klinisches Risiko) ist in Abbildung 3 wiedergegeben.

Hämodynamisch instabile Patienten werden intensivmedizinisch überwacht; alle kreislauf- und atmungsunterstützenden Maßnahmen müssen sofort verfügbar sein. Die sofortige Therapie ist für die Prognose entscheidend. Jeglicher Zeitverzug für aufwändige Diagnostik und Transporte ist zu vermeiden.

Die CTPA ist aktuell der diagnostische Goldstandard zur Bestätigung oder zum Ausschluss einer klinisch vermuteten LE. Dies gilt grundsätzlich auch für hämodynamisch instabile Patienten. Falls die CTPA jedoch nicht sofort verfügbar oder - aufgrund ausgeprägter Instabilität und unmittelbarer Lebensgefahr - nicht umgehend durchführbar ist, hat die transthorakale Echokardiographie in dieser Notfallsituation einen hohen diagnostischen Wert (Abb. 2). Bei zugrundeliegender LE ist die akute rechtsventrikuläre (RV) Dysfunktion als Ursache der klinischen Instabilität anzusehen; sie ist in aller Regel leicht und zuverlässig echokardiographisch erkennbar. Bei fehlender RV-Dysfunktion kann eine massive LE als Ursache der klinischen Instabilität ausgeschlossen werden und es ist nach einer alternativen Diagnose zu suchen. Wenn jedoch Zweifel bestehen, dass es sich bei der echokardiograpisch festgestellten RV-Dysfunktion um ein akutes Ereignis handelt (wie z.B. bei bekannter chronischer Lungenerkrankung oder chronisch-thromboembolischer pulmonaler Hypertension) oder wenn aus technischen Gründen kein aussagekräftiger Befund erhoben werden kann (z.B. Emphysem, extreme Adipositas), müssen die Patienten bei fortbestehendem Verdacht auf LE einer weiteren bildgebenden Diagnostik unterzogen werden. In den meisten anderen Fällen - und insbesondere bei fehlender Durchführbarkeit einer CTPA aufgrund persistierender hämodynamischer Instabilität - gilt die Kombination aus klinischer 


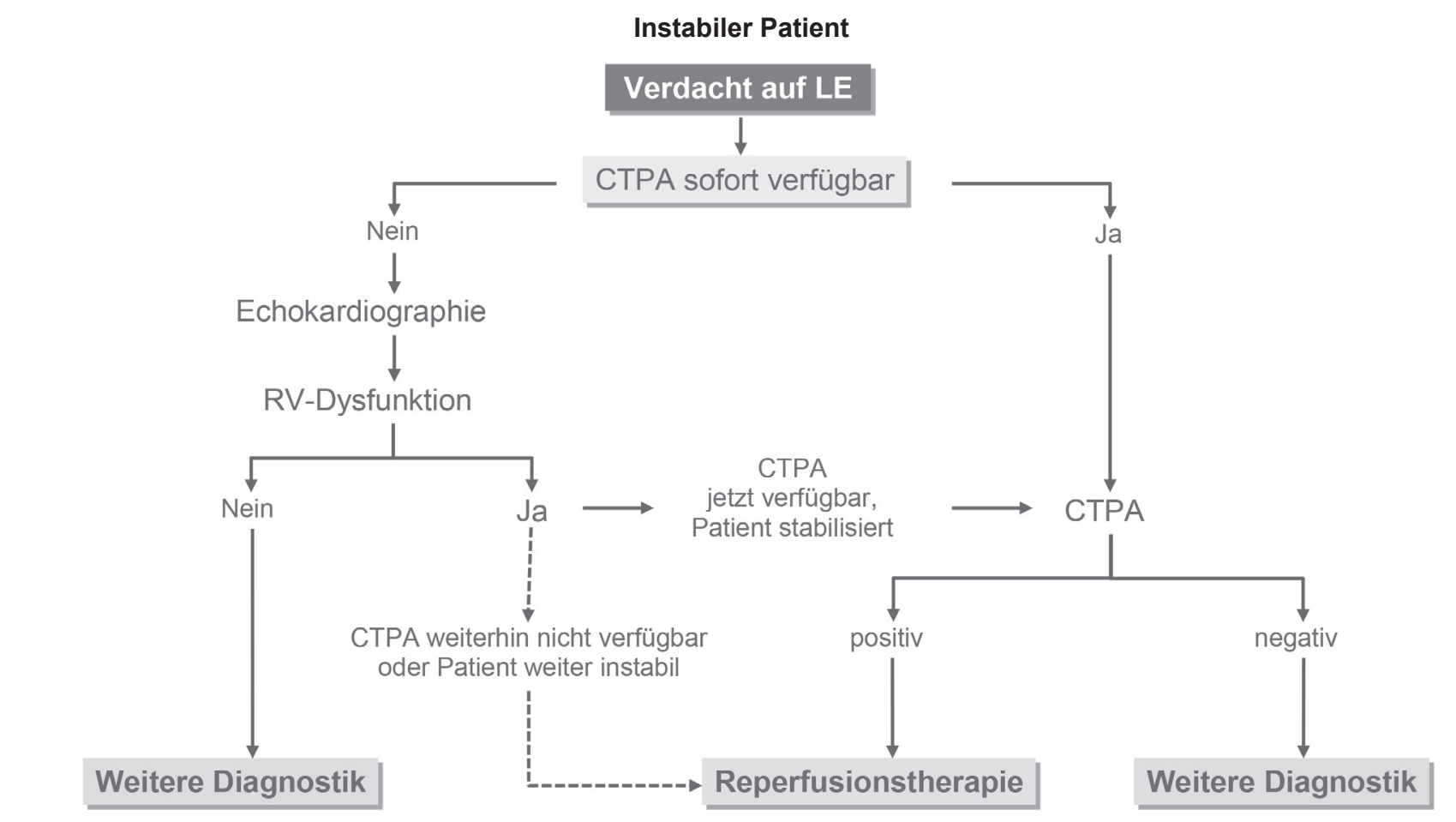

CTPA = computertomographische Pulmonalisangiographie, $\mathrm{LE}=$ Lungenembolie, $\mathrm{RV}=$ rechtsventrikulär

Abbildung 2: Diagnostischer Algorithmus: Verdacht auf Lungenembolie mit hohem klinischen Risiko - instabiler Patient (modifiziert nach [151])

Instabilität und RV-Dysfunktion im Echokardiogramm als beweisend für die Diagnose einer massiven LE; das erfordert dann die unverzügliche Einleitung der Therapie.

Bei hämodynamisch stabilen Patienten hat das diagnostische Vorgehen anhand eines Algorithmus zum Ziel, aus der Gruppe der Verdachtsfälle die Minderheit der Patienten mit LE sicher zu identifizieren und der Mehrheit der Patienten ohne diese Erkrankung weitere diagnostische Verfahren mit den damit verbundenen Risiken zu ersparen. Die Kriterien, nach denen ein Algorithmus als ausreichend sicher beurteilt wird, sind im Kapitel „Bein- und Beckenvenenthrombose" beschrieben. Die eingesetzten Untersuchungsverfahren sind von den lokalen Gegebenheiten abhängig [152].

Bei Verdacht auf Lungenembolie unterscheidet sich die diagnostische Vorgehensweise bei einem klinisch stabilen Patienten (nicht hohes Risiko) grundsätzlich von jener bei einem instabilen Patienten (hohes Risiko). Das Kriterium für Stabilität ist in erster Linie die Hämodynamik (Puls, arterieller Blutdruck, klinische Zeichen der verminderten Organperfusion).

Für den stabilen Patienten wurden verschiedene diagnostische Algorithmen vorgeschlagen und getestet. Unter Berücksichtigung der aktuellen Datenlage kann heute folgendes Vorgehen empfohlen werden (Abb. 3):

Der erste Diagnoseschritt sollte die Bestimmung der klinischen Wahrscheinlichkeit sein. Patienten mit niedriger und mittlerer/ intermediärer klinischer Wahrscheinlichkeit, die im Weiteren einen negativen D-Dimer-Test aufweisen, bedürfen keiner weiterführenden (bildgebenden) Diagnostik; eine Lungenembolie gilt als ausgeschlossen [302]. Bei
Patienten mit hoher klinischer Wahrscheinlichkeit folgen von vornherein, also ohne Bestimmung des D-Dimer-Tests, weitere bildgebende Untersuchungen. Für Patienten mit nicht hoher (niedriger/ mittlerer) klinischer Wahrscheinlichkeit und positivem D-Dimer-Test ist ebenfalls eine weiterführende Diagnostik erforderlich.

Im zweiten Diagnoseschritt sollten bildgebende Verfahren angewendet werden. Deren Auswahl hängt von der lokalen Verfügbarkeit ab. Dabei sollte bei minimaler Belastung des Patienten und geringem Verbrauch von Ressourcen ein Maximum an diagnostischer Aussagekraft erzielt werden. Unter Berücksichtigung der in den letzten 10 Jahren publizierten Diagnose- und Management-Studien [217, 272, 288] sowie der klinischen Praktikabilität wird in Deutschland sowie in den meisten anderen europäischen Ländern der in Abbildung 3 dargestellte diagnostische Algorithmus empfohlen, der in der Regel auf der CTPA als primärer bildgebender Methode basiert. Das schließt allerdings die Validität anderer bildgebender Untersuchungen keineswegs aus. So sollte z.B. die Ventilations-Perfusionsszintigraphie der Lungen insbesondere dann eingesetzt werden, wenn die CTPA bei individueller Abschätzung zu risikoreich erscheint (schwere Niereninsuffizienz, schwere Jod-Kontrastmittelallergie oder ggf. in der Schwangerschaft). Wenn eine qualifizierte Sonographie der Beinvenen zeitnah zur Verfügung steht, kann damit ein gewisser Anteil der Patienten mit venöser Thromboembolie identifiziert werden; bei nachgewiesener Beinvenenthrombose sind dann keine weiteren Untersuchungen erforderlich, um eine therapeutische Entscheidung zu treffen. Auch der Lungenultraschall kann bei ent- 


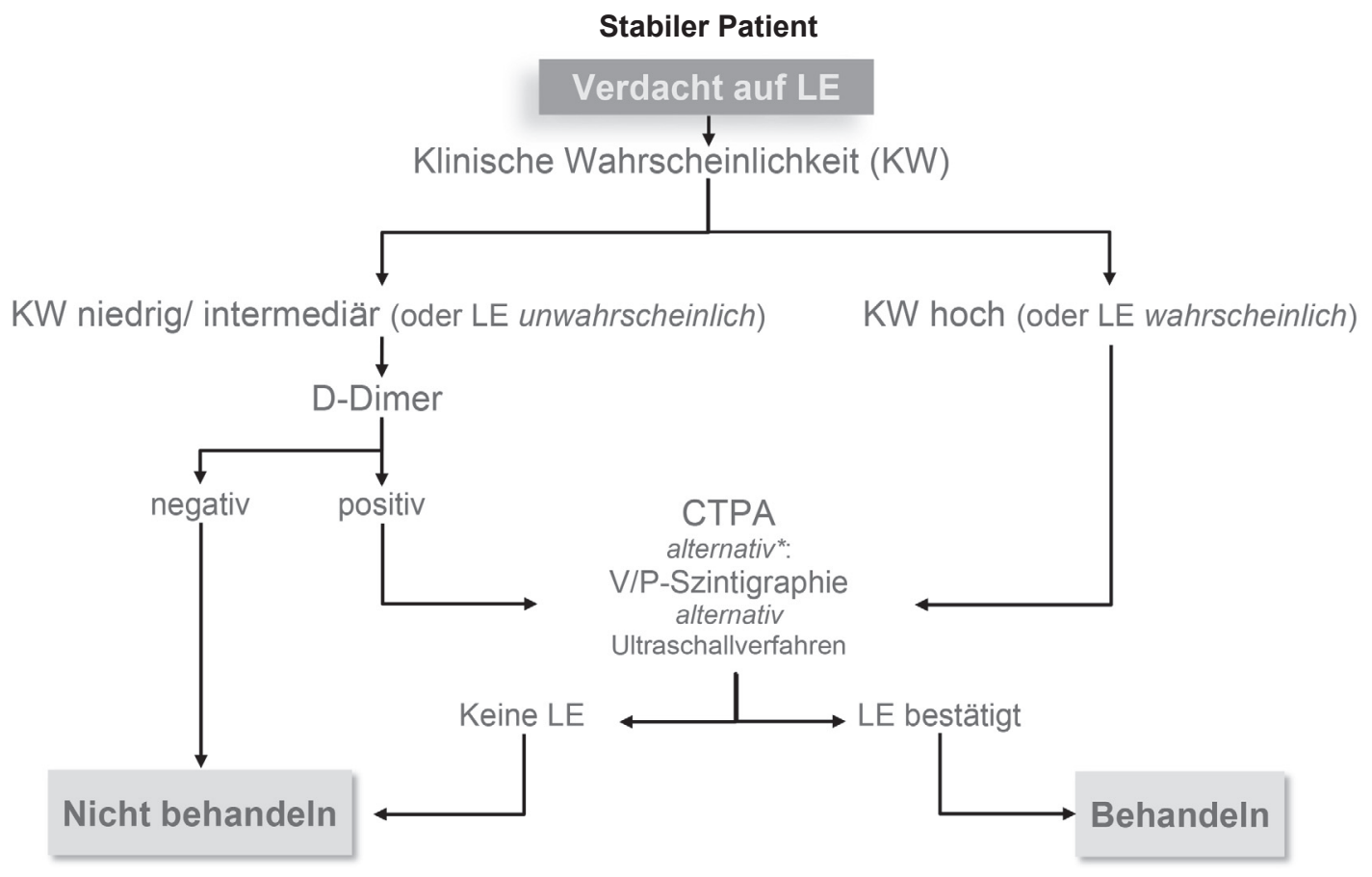

CTPA = Computertomographische Pulmonalisangiographie, $\mathrm{KW}=$ klinische Wahrscheinlichkeit, $\mathrm{V} / \mathrm{P}=$ Ventilations-/Perfusions-Szintigraphie

* Alternative Verfahren sind insbesondere indiziert, wenn die CTPA bei individueller Abschätzung zu risikoreich erscheint. Weniger invasiv ist die V/P-

Szintigraphie und nicht-invasiv sind die Ultraschallverfahren. Bei sonographischem Nachweis einer Beinvenenthrombose kann der Verdacht auf LE als

bestätigt angesehen werden, denn die Therapie ist identisch. Der Lungenultraschall kann zum LE-Nachweis mit herangezogen werden. Bei negativen

Ultraschallbefunden ist eine LE nicht ausgeschlossen!

Abbildung 3: Diagnostischer Algorithmus: Verdacht auf Lungenembolie ohne hohes klinisches Risiko - stabiler Patient (modifiziert nach [151])

Tabelle VI a: Validierte klinische Scores zur Risikostratifizierung einer nachgewiesenen Lungenembolie: Original-PESI und SPESI: klinische Parameter [151]

\begin{tabular}{|c|c|c|}
\hline \multirow{2}{*}{ Klinische Parameter } & \multicolumn{2}{|c|}{ Punkte } \\
\hline & Original-PESI & sPESI \\
\hline Lebensalter & Alter in Jahren & $\begin{array}{c}1 \text { Punkt } \\
\text { wenn Alter } \\
>80 \text { Jahre }\end{array}$ \\
\hline Männliches Geschlecht & +10 & - \\
\hline Tumorerkrankung & +30 & 1 \\
\hline Chronische Herzinsuffizienz & +10 & 1 \\
\hline Chronische Lungenerkrankung & +10 & \\
\hline $\begin{array}{l}\text { Pulsfrequenz } \geq 110 \text { Schläge } \\
\text { pro Minute }\end{array}$ & +20 & 1 \\
\hline $\begin{array}{l}\text { Systolischer Blutdruck } \\
<100 \mathrm{mmHg}\end{array}$ & +30 & 1 \\
\hline $\begin{array}{l}\text { Atemfrequenz > } 30 \text { Atemzüge } \\
\text { pro Minute }\end{array}$ & +20 & - \\
\hline Temperatur $<36^{\circ} \mathrm{C}$ & +20 & - \\
\hline Bewusstseinsstörung & +60 & - \\
\hline $\begin{array}{l}\text { Arterielle Hämoglobin- } \\
\text { sättigung }<90 \%\end{array}$ & +20 & 1 \\
\hline
\end{tabular}

PESI = Pulmonary Embolism Severity Index; sPESI = simplified PESI. Beispiel: 60-jähriger Mann, ohne Tumorerkrankung, Herzfrequenz 129 Schläge/min, Normalwerte für Blutdruck und Temperatur erhält im PESI-Score 90 Punkte) $[60$ (Alter) +10 (Geschlecht) +20 (Tachykardie)] und im sPESI-Score 1 Punkt (Tachykardie) sprechender Expertise zur Abklärung des Verdachts auf LE eingesetzt werden.

\section{Therapie der Lungenembolie}

\section{Risikostratifizierung}

Bereits bei Verdacht auf eine Lungenembolie (LE) unterscheidet sich die diagnostische Vorgehensweise bei einem klinisch stabilen Patienten (= nicht hohes Risiko) grundsätzlich von jener bei einem instabilen Patienten (= hohes Risiko). Während Patienten mit kardiogenem Schock oder persistierender Hypotension vom Anfang an intensivmedizinisch überwacht und behandelt werden sollen, ist bei hämodynamisch stabilen Patienten nach Bestätigung der Diagnose als nächster Schritt eine weitere Risikostratifizierung indiziert, um eine an den klinischen und hämodynamischen Schweregrad der LE angepasste Therapie zu ermöglichen.

Die Risikoabschätzung beim hämodynamisch stabilen Patienten mit nachgewiesener Lungenembolie beginnt mit einem validierten klinischen prognostischen Score, beispielsweise dem Pulmonary Embolism Severity Index im Original (= PESI) oder in der vereinfachten Version (= sPESI) (Tab. VI a und VI b). Der PESI berücksichtigt und integriert demographische Faktoren (Alter und Geschlecht), Komorbidität (kardiale und pulmonale Vorerkrankungen, Malignom) und klinische Befunde bei Diagnosestellung (Blutdruck, Puls, Atemfrequenz, Temperatur, Vigilanz, ar- 
terielle Sauerstoffsättigung). Etwa ein Drittel aller Patienten mit LE sind in der PESI-Kategorie I-II bzw. haben einen sPESI von 0. Bei diesen Patienten besteht - unter der Voraussetzung einer adäquaten Antikoagulation - ein niedriges Risiko für einen ungünstigen Verlauf in der Akutphase $[15,130]$; (s. Tab. VII).

Patienten in der PESI-Kategorie III-V oder jene mit einem sPESI von $>1$ haben eine 30 -Tages-Mortalität von mindestens $11 \%$ und somit ein intermediäres klinisches Risiko [15, 130]; (Tabellen VI a und VI b). Dabei definiert das Vorliegen einer RV-Dysfunktion (in der CTPA oder in der Echokardiographie) in Kombination mit einem oder beiden positiven kardialen Biomarkern (Troponin-Test, natriuretische Peptide) ein intermediär-hohes klinisches Risiko (Tab. VII); bei diesen Patienten ergibt sich die Indikation zur Überwachung und ggf. zur Reperfusionstherapie. Patienten mit allen anderen Befundkombinationen (keine RV-Dysfunktion und/oder kardiale Biomarker negativ) sind dagegen in die Kategorie des intermediär-niedrigen klinischen Risikos einzustufen; hier wird wegen der guten Prognose ausschließlich die Antikoagulation empfohlen.

\section{Antikoagulation}

Für die Mehrzahl der Patienten mit Lungenembolie gelten bezüglich der initialen Antikoagulation, der Erhaltungstherapie und der verlängerten Erhaltungstherapie dieselben Empfehlungen wie für die Beinvenenthrombose (s. „Therapie der Beinvenenthrombose“). Ein wesentlicher Unterschied besteht für Patienten mit hämodynamisch instabiler Lungenembolie, die eine medikamentöse Thrombolyse erhalten sollen; in diesen Fällen wird initial Heparin verabreicht, bevorzugt unfraktioniertes Heparin (UFH) als Bolus.

Die sofortige Antikoagulation mit Heparin senkt die Morbidität und die Mortalität bei einer Lungenembolie; sie sollte deshalb bei hoher klinischer Wahrscheinlichkeit noch vor apparativer Bestätigung der Diagnose eingeleitet werden [143]. Die Effektivität von UFH wurde bereits 1960 unter Beweis gestellt [19]. Es liegen zwei randomisierte Studien vor, die die initiale Antikoagulation der hämodynamisch stabilen Lungenembolie mit niedermolekularem Heparin (NMH) bzw. mit Fondaparinux untersucht und deren gleich gute Wirksamkeit und Sicherheit im Ver-
Tabelle VI b: Validierte klinische Scores zur Risikostratifizierung einer nachgewiesenen Lungenembolie: Original-PESI und sPESI: Risikokategorien [151]

\begin{tabular}{ll}
\hline \multicolumn{2}{c}{ Risikokategorien } \\
\hline Original-PESI & sPESI \\
\hline Kategorie I: $\leq 65$ Punkte & 0 Punkte: \\
$\begin{array}{l}\text { sehr niedriges 30-Tages-Todes- } \\
\text { risiko }(0-1,6 \%)\end{array}$ & $\begin{array}{l}30 \text {-Tages-Todesrisiko: 1,0 \% } \\
(95 \% \mathrm{VI}: 0,0-2,1 \%)\end{array}$
\end{tabular}

Kategorie II: 66-85 Punkte

niedriges 30-Tages-Todesrisiko

$(1,7-3,5 \%)$

Kategorie III: 86-105 Punkte
moderates 30-Tages-Todes-

risiko $(3,2-7,1 \%)$

$\geq 1$ Punkt

30-Tages-Todesrisiko: 10,9\%

(95 \% VI : 8,5-13,2\%)

Kategorie IV: 106-125 Punkte

hohes Todesrisiko $(4,0-11,4 \%)$

Kategorie V: >125 Punkte

sehr hohes Todesrisiko

$(10,0-24,5 \%)$

PESI = Pulmonary Embolism Severity Index; sPESI = simplified PESI; $95 \% \mathrm{VI}=95 \%$ Vertrauensintervall

gleich zu UFH belegt haben $[40,266]$. Es ist anzunehmen, dass dieser Sachverhalt für die gesamte Gruppe der $\mathrm{NMH}$ zutrifft [196, 303].

Die initiale Antikoagulation mit Heparin oder Fondaparinux sollte - analog zur Beinvenenthrombose - mindestens 5 Tage erfolgen. Bei Erhaltungstherapie mit einem Vitamin K-Antagonist (VKA), wird die initiale Antikoagulation so lange beibehalten, bis eine INR >2,0 über mindestens 24 Stunden erreicht wurde [143]. Wenn für die Erhaltungstherapie Dabigatran oder Edoxaban vorgesehen sind, wird nach der initialen Gabe von UFH, NMH oder Fondaparinux ohne Überlappung ab Tag 5, 6 oder 7 mit dem oralen Antikoagulans weiter therapiert. Soll der Patient mit Apixaban oder Rivaroxaban behandelt werden, kann direkt nach Diagnosestellung mit diesen Medikamenten begonnen werden, allerdings mit einer erhöhten Anfangsdosis für 1 bzw. 3 Wochen [3, 22, 41, 42, 256, 257]; für Einzelheiten s. „Therapie der Beinvenenthrombose“ und Tabelle II.

Tabelle VII: Risikostratifizierung der Lungenembolie [151]

\begin{tabular}{|c|c|c|c|c|c|}
\hline \multicolumn{2}{|c|}{ Klinisches Risiko } & \multicolumn{4}{|c|}{ Risikoparameter und Scores } \\
\hline & & $\begin{array}{l}\text { Schock oder } \\
\text { Hypotension }\end{array}$ & $\begin{array}{l}\text { PESI-Kategorie III-V } \\
\text { oder sPESI } \geq 1\end{array}$ & $\begin{array}{l}\text { RV- Dysfunktion } \\
\text { (Bildgebung*) }\end{array}$ & $\begin{array}{l}\text { Kardiale biochemische } \\
\text { Marker** }\end{array}$ \\
\hline Hoch & & + & $(+)$ & + & $(+)$ \\
\hline \multirow[t]{2}{*}{ Intermediär } & Intermediär-hoch & - & + & \multicolumn{2}{|c|}{ Beide positiv } \\
\hline & Intermediär-niedrig & - & + & \multicolumn{2}{|c|}{ Ein (oder kein) Kriterium positiv } \\
\hline Niedrig & & - & - & \multicolumn{2}{|c|}{$\begin{array}{c}\text { Bestimmung optional; } \\
\text { falls bestimmt: beide negativ }\end{array}$} \\
\hline
\end{tabular}

PESI = Pulmonary Embolism Severity Index; sPESI = simplified PESI; RV = rechtsventrikuläre Dysfunktion;

* Bildgebung = Echokardiographie oder CT; ** Kardiale biochemische Marker = Troponin, natriuretische Peptide 


\section{Thrombolyse}

Als Reperfusionsmaßnahmen kommen bei akuter Lungenembolie (LE) die systemische Thrombolyse und verschiedene mechanische Verfahren in Betracht. Die Wirksamkeit der systemischen Thrombolyse ist zweifelsfrei belegt [181]. Dafür stehen Streptokinase, Urokinase und rekombinanter Gewebe-Plasminogenaktivator (rt-PA) zur Verfügung (s. Tab. VIII). Andere Thrombolytika wurden in Studien erfolgreich getestet, sind jedoch zum jetzigen Zeitpunkt nicht für diese Indikation zugelassen.

Die systemische Thrombolyse führt binnen kurzer Zeit zu einer Reduktion der Thrombusmasse in der pulmonalarteriellen Strombahn und entlastet damit den rechten Ventrikel [13, 63]. Andererseits ist die Thrombolyse mit einem erheblichen Risiko größerer, insbesondere intrakranieller, Blutungen verbunden; letztere treten in ca. 2\% der thrombolytisch behandelten Patienten auf [149, 192]. Aus diesem Grund ist die Thrombolyse - als Primärtherapie - grundsätzlich nur bei hämodynamisch instabilen $\mathrm{Pa}$ tienten indiziert, da diese ein hohes Risiko von LE-bedingtem Tod oder lebensbedrohlichen Komplikationen in den ersten Stunden oder Tagen nach Diagnose haben. Die Nutzen-Risiko-Abwägung einer thrombolytischen Behandlung bei hämodynamisch stabilen Patienten mit intermediär-hohem Risiko wird weiter unten diskutiert (s. „Risiko-adaptierte therapeutische Strategie“).

Bei Patienten mit flottierenden Thromben in den rechten Herzhöhlen bleibt der Stellenwert einer thrombolytischen Behandlung als Alternative zur chirurgischen Embolektomie kontrovers. Während in manchen Patientenkohorten gute Ergebnisse mittels Thrombolyse erzielt werden konnten $[86,219]$, berichteten andere Studien übereine hohe Letalität von über $20 \%$ in der Akutphase $[52,286]$.

\section{Mechanische Reperfusionsverfahren}

Bis zu zwei Dritteln aller Patienten mit Lungenembolie (LE) und hämodynamischer Instabilität (hohes Risiko) bleibt eine systemische Thrombolyse vorenthalten [174, 269]. Teilweise liegt dies an dem im Vergleich zur alleinigen Heparintherapie erhöhten Risiko an intrakraniellen $(2,4 \%$ vs. $0,2 \% ; p<0,001)$ und großen, nicht-intrakraniellen $(6,3 \%$ vs. 1,5\%) Blutungen [192]. Als Alternativen stehen, insbesondere für Patienten mit hohem Blutungsrisiko, die opera- tive Thrombektomie und die kathetergestützten Verfahren zur Verfügung.

Kathetergestützte Verfahren können mit oder ohne gleichzeitige Thrombolyse durchgeführt werden; Thrombolytika werden dabei meist niedrig dosiert, mitunter auch zusätzlich ultraschallassistiert verabreicht [78]. Die mechanischen Verfahren arbeiten mittels Rotations-, Aspirations-, hydrodynamischer oder Saug-Thrombektomie; das Ziel besteht darin, über eine Verkleinerung der Thrombusgröße und Reduktion der Thrombuslast die rechtsventrikuläre Funktion zu verbessern. Eine Übersicht verschiedener kathetergestützter Verfahren aus 6 prospektiven und 29 retrospektiven, nicht-randomisierten Studien zeigte eine klinische Erfolgsrate von insgesamt 87\%, definiert als Kombination aus hämodynamischer Stabilisierung, Verbesserung der Hypoxämie und Überleben bis zum Entlassungszeitpunkt [155]; angemerkt werden sollte, dass $67 \%$ der Patienten zusätzlich eine lokale Thrombolyse erhielten.

Eine Erhöhung des enddiastolischen Durchmessers von rechtem gegenüber linkem Ventrikel über 0,9 bis 1,0 bei akuter Lungenembolie gilt als validierter prognostischer Parameter in der Akutphase [87, 252]. Während Heparin nur einen geringen Effekt auf die Verbesserung der R/VFunktion in den ersten 48 Stunden hat [150], scheint der Effekt einer kathetergestützten, niedrig dosierten Thrombolyse dem einer systemischen Thrombolyse vergleichbar zu sein [80].

Die einzige bis heute publizierte prospektive randomisierte Studie, die ein kathetergestütztes Verfahren (ultraschallassistierte lokale Thrombolyse mittels $10 \mathrm{mg}$ rt-PA pro betroffener Lunge über 15 Stunden) gegen unfraktioniertes Heparin bei LE-Patienten mit intermediärem Risiko verglich, zeigte eine signifikante Verbesserung des primären Surrogatendpunkts RV-Dysfunktion innerhalb 24 Stunden in der kathetergestützt behandelten Patientengruppe gegenüber den allein mit Heparin behandelten Patienten [153].

Die kathetergestützten Verfahren stellen damit bei Patienten mit massiver LE und rechtsventrikulärer Dysfunktion eine therapeutische Alternative dar, insbesondere bei Kontraindikationen gegen eine systemische Thrombolyse, bei indizierter Reperfusion und gleichzeitig erhöhtem Blutungsrisiko, bei unzureichendem Erfolg

Tabelle VIII: Validierte Thrombolyse-Schemata zur Therapie der akuten Lungenembolie [158]

\begin{tabular}{|c|c|}
\hline Alteplase (rt-PA) & $\begin{array}{l}\text { Bolus-Injektion von } 10 \text { mg über } 1 \text { - } 2 \text { min, gefolgt von } 90 \text { mg über } 2 \mathrm{~h} \\
\text { oder } 100 \text { mg über } 2 \mathrm{~h} \\
\text { oder akzeleriert: } 0,6 \mathrm{mg} / \mathrm{kg} \text { über } 15 \mathrm{~min}\end{array}$ \\
\hline Streptokinase & $\begin{array}{l}250.000 \text { IE über } 30 \text { min, gefolgt von } 100.000 \text { IE/h über } 12-24 \mathrm{~h} \\
\text { oder akzeleriert: } 1,5 \mathrm{Mio} \text {. IE über } 2 \mathrm{~h}\end{array}$ \\
\hline Urokinase & $\begin{array}{l}4.400 \text { IE/kg KG über } 10 \text { min, gefolgt von } 4.400 \mathrm{IE} / \mathrm{kg} / \mathrm{h} \text { über } 12-24 \mathrm{~h} \\
\text { oder akzeleriert: } 3 \text { Mio. IE über } 2 \mathrm{~h}\end{array}$ \\
\hline Reteplase & $\begin{array}{l}\text { Zwei Bolus-Injektionen à } 10 \text { IU im Intervall von } 30 \text { min } \\
\text { (in einer prospektiven Studie validiert; noch keine Zulassung für diese Indikation) }\end{array}$ \\
\hline Tenecteplase & $\begin{array}{l}\text { Gewichtsadaptiertes Schema mit Bolus-Injektion von } 30 \text { - } 50 \text { mg über } 5 \text { - } 10 \text { sec wie beim akuten Myokardinfarkt (in } \\
\text { einer prospektiven Studie validiert: noch keine Zulassung für diese Indikation) }\end{array}$ \\
\hline
\end{tabular}


einer bereits durchgeführten Thrombolyse sowie bei indizierter, jedoch nicht verfügbarer operativer Thrombektomie [73].

\section{Vena cava-Filter}

Die Indikation zur Implantation eines Vena cava-Filters bei Lungenembolie bleibt auf wenige Ausnahmesituationen beschränkt (s. „Vena Cava-Filter" im Abschnitt Beinvenenthrombose).

\section{Risiko-adaptierte therapeutische Strategie}

Für die Frage, ob bei der Therapie der akuten Lungenembolie eine alleinige Antikoagulation ausreicht oder ob zusätzliche Maßnahmen eingesetzt werden müssen, ist primär die hämodynamische Stabilität des Patienten ausschlaggebend.

Patienten mit symptomatischer Lungenembolie sollen therapeutisch antikoaguliert werden.

Hämodynamisch instabile Patienten sollen eine sofortige Reperfusionstherapie erhalten.

Hämodynamisch stabile Patienten mit intermediär-hohem Risiko sollten nur dann eine Reperfusionstherapie erhalten, wenn sich im Verlauf Zeichen einer hämodynamischen Dekompensation einstellen.

Patienten, die für eine Reperfusionstherapie in Frage kommen, sollten initial mit unfraktioniertem oder niedermolekularem Heparin behandelt werden.

Bei hämodynamisch instabilen Patienten (hohes Risiko) sollte die systemische Thrombolyse zur Anwendung kommen, vorzugsweise mit rekombinantem Gewebeplasminogen-Aktivator (rt-PA). Vor der Thrombolyse sowie im Anschluss daran, erfolgt die begleitende Antikoagulation mit Heparin, bevorzugt mit unfraktioniertem Heparin (UFH). Die klinische Situation ist entscheidend, wenn es darum geht, in welchem Ausmaß Kontraindikationen der Thrombolyse berücksichtigt werden können. Als Alternativen sind bei absoluten Kontraindikationen zur Thrombolyse (z.B. bei sehr hohem Blutungsrisiko) die kathetergestützte Thrombusfragmentation mit oder ohne niedrig dosierte lokale Thrombolyse bzw. die chirurgische Pulmonalisembolektomie unter extrakorporaler Zirkulation zu diskutieren.

Patienten mit niedrigem oder intermediär-niedrigem Risiko sollen antikoaguliert werden wie Patienten mit alleiniger Beinvenenthrombose.

Bei Patienten mit niedrigem Risiko kann die Behandlung der Lungenembolie ambulant erfolgen.

Relativ einfach ist die therapeutische Entscheidung bei Patienten mit Reanimationspflicht. Wegen der infausten Prognose ohne rekanalisierte pulmonalarterielle Strombahn gelten keine Kontraindikationen der Thrombolyse. Wenn jedoch bei offensichtlichen Kontraindikationen zur systemischen Thrombolyse mechanische Reperfusions- verfahren unmittelbar zur Verfügung stehen, kann deren Einsatz alternativ erwogen werden. Die Reanimation sollte in der Regel über mindestens 60 Minuten durchgeführt werden.

Patienten mit intermediär-hohem Risiko (Tab. VII), d. h. ohne kardiogenen Schock oder persistierende Hypotension, aber mit einem hohen PESI-Score und rechtsventrikulärer Dysfunktion sowie positiven kardialen Biomarken, sollten initial mit Heparin antikoaguliert und über mindestens 2 bis 3 Tage intensivmedizinisch überwacht werden, da das Risiko einer potenziell tödlichen hämodynamischen Dekompensation innerhalb der ersten 7 Tage fast $6 \%$ beträgt [192]. In einer großen randomisierten Studie konnte zwar eine primäre systemische Thrombolyse das frühe Todes- und Komplikationsrisiko dieser Patienten signifikant reduzieren, die Inzidenzrate intrakranieller Blutungen war allerdings mit $2 \%$ hoch [192]. Daher sollte eine Thrombolyse nur als "Rescue"-Verfahren, d.h. bei Auftreten einer hämodynamischen Dekompensation im Verlauf durchgeführt werden. Als Dekompensationskriterien gelten: 1) Kreislaufkollaps mit Reanimationspflicht, 2) systolischer Blutdruckabfall auf $<90 \mathrm{mmHg}$ bzw. um mindestens 40 $\mathrm{mmHg}$ über $>15 \mathrm{~min}$, der von klinischen Zeichen einer verminderten Organperfusion (kalte Extremitäten, Urinausscheidung $<30 \mathrm{ml} / \mathrm{h}$, Vigilanzstörung oder Verwirrung) begleitet wird, 3) Katecholaminbedarf (außer Dopamin mit einer Infusionsrate $<5 \mu \mathrm{g} / \mathrm{kg} / \mathrm{min}$ ) um einen systolischen Druck von $>90 \mathrm{mmHg}$ und eine adäquate Organperfusion aufrecht zu erhalten [192, 271]. Als alternative Reperfusionsverfahren kommen - insbesondere bei hohem Blutungsrisiko und anderen Kontraindikationen zur systemischen Thrombolyse - eine kathetergestützte, ggf. ultraschallassistierte, niedrig dosierte lokale Thrombolyse [80] oder die chirurgische Pulmonalisembolektomie in Frage.

Bei hämodynamisch stabilen Patienten mit intermediärniedrigem oder niedrigem Risiko ist die alleinige Antikoagulation die Therapie der Wahl (siehe "Erhaltungstherapie“ und "Verlängerte Erhaltungstherapie“ im Abschnitt Beinvenenthrombose sowie Tab. II). Bei diesen Patienten ist keine Immobilisierung erforderlich.

Die ambulante Betreuung eines Patienten mit akuter Lungenembolie kommt allenfalls bei einem niedrigen Risiko in Betracht. Die Entscheidung hängt von mehreren Faktoren ab, u.a. der lokalen ärztlichen und häuslichen Betreuung. Bei günstigen Voraussetzungen erscheint eine frühe Entlassung (sofort oder binnen 48 Stunden) unter Fortführung der Antikoagulation unter ambulanten Bedingungen effektiv und sicher [314].

Bei Patienten mit Lungenembolie liegt in 90 \% der Fälle gleichzeitig eine Bein- und / oder Beckenvenenthrombose vor. Sobald die mit der LE einhergehende akute Gefährdung des Patienten überwunden ist, sollte eine Untersuchung des peripheren Venensystems erfolgen. Die Indikation zu Notwendigkeit und Dauer einer Kompressionstherapie ergibt sich aus dem Thrombosebefund an den Beinvenen. 


\section{Besondere Krankheitsentitäten}

Lungenembolie in Schwangerschaft und Wochenbett

Die Diagnostik bei Verdacht auf Lungenembolie (LE) in Schwangerschaft und Wochenbett wirft spezielle Probleme auf, denn es gibt keinen unter diesen Bedingungen getesteten Algorithmus.

In der Schwangerschaft soll jeder Verdacht auf Lungenembolie umgehend soweit abgeklärt werden, dass eine therapeutische Entscheidung erfolgen kann; das schließt auch strahlendiagnostische Methoden ein.

Die klinische Wahrscheinlichkeit ist - allein durch die vorliegende Schwangerschaft - höher als bei nicht Schwangeren. Der D-Dimer-Test ist wegen des physiologischen Anstiegs der D-Dimere in der Schwangerschaft nur eingeschränkt verwertbar; allerdings existieren an das Schwangerschaftsalter adaptierte Referenzwerte, die die Interpretation erleichtern [198]. Die mit einer Strahlenexposition einhergehenden Verfahren sind vor allem in den ersten beiden Dritteln der Schwangerschaft kritisch zu prüfen; deren Einsatz ist aber unter Würdigung des klinischen Zustandes gerechtfertigt, um eine LE definitiv auszuschließen bzw. nachzuweisen. Auch unter dem Aspekt der Strahlenexposition erscheint es rational, bei primärem Verdacht auf LE - und unter der Voraussetzung hämodynamischer Stabilität - zunächst eine bilaterale Sonographie der Bein- und Beckenvenen durchzuführen (gegebenenfalls in Kombination mit Lungenultraschall, falls entsprechende Expertise verfügbar ist) und bei Thrombosenachweis ohne spezielle LE-Diagnostik zu therapieren. Bei negativem Befund besteht die Wahl zwischen CTPA und Szintigraphie.

Die Strahlenexposition der Mammae ist bei der Szintigraphie deutlich niedriger als bei der CTPA, hinsichtlich der fötalen Dosis scheint dagegen der Unterschied nicht signifikant zu sein [201]. Bei Schwangeren liegen in der Regel keine strukturellen Lungenveränderungen vor (COPD o.ä.), so dass bei der Szintigraphie meistens eine Perfusions-Szintigraphie in SPECT-Technik ausreichend ist (applizierte Aktivität ca. 40 MBq 99mTc-MAA). Die fötale Strahlenexposition beträgt dann in der Regel nicht mehr als 0,2 mSv [245], und die maternale Strahlenexpo- sition liegt unter 0,8 mSv. Bei unklarem Befund kann die Ventilationsuntersuchung nachgeholt werden, was dann aber die Strahlenexposition erhöht (Tab. IX).

Bei der CTPA sollten alle Möglichkeiten der technischen Dosisoptimierung eingesetzt werden (niedrige $\mathrm{kVp}$, mAs -Anpassung an das Körpergewicht, Dosismodulationsprogramme, ultraschnelle Datenakquisition, iterative Rekonstruktion), um insbesondere die Dosis der Mammae zu minimieren [187]. Die Strahlenbelastung für den Fötus selbst ist wie bei der Perfusionsszintigraphie nahezu vernachlässigbar, da er nicht im direkten Scangebiet liegt (Tabelle IX).

Prinzipiell kann mit einer nicht kontrastmittel-gebundenen MR-Angiographie jegliche Strahlenexposition vermieden werden. Das Verfahren ist für diese Fragestellung jedoch an eine hohe lokale Expertise gebunden und methodisch nicht abschließend validiert [53, 64, 148, 236].

Die Therapie einer Lungenembolie in der Schwangerschaft sollte initial stationär erfolgen. Bei hämodynamisch stabilem Zustand wird die Antikoagulation analog zur Venenthrombose durchgeführt. Bei hämodynamisch instabilem Zustand und lebensbedrohlicher Situation kommen eine Thrombolyse oder mechanische Verfahren in Betracht; für die Schwangerschaft liegen hierzu jedoch nur Einzelfallberichte vor. Thrombolytika passieren nicht die Plazenta, erhöhen aber dennoch das Blutungsrisiko für Mutter und Kind und bedürfen daher einer besonders sorgfältigen Nutzen-Risiko-Abwägung [50].

Eine in der Schwangerschaft aufgetretene LE bedarf während der gesamten Schwangerschaft sowie darüber hinaus in der postpartalen Phase einer Antikoagulation, mindestens aber für einen Gesamtzeitraum von 3 Monaten.

Vena cava-Filter sind sehr selten erforderlich; wenn überhaupt, sollten passagere Filter eingesetzt werden. Eine Indikation kann die unmittelbar präpartal auftretende schwere LE sein bzw. die Notwendigkeit, eine indizierte hochdosierte Antikoagulation wegen schwerer Blutungskomplikationen [50] oder wegen der unmittelbar bevorstehenden Geburt pausieren zu müssen.

\section{Inzidentelle / asymptomatische Lungenembolie}

Der „zufällige“ - ohne Vorliegen eines expliziten klinischen Verdachts - computertomographische Nachweis ei-

Tab. IX: Geschätzte Strahlenbelastung radiologischer Methoden bei der Abklärung einer Lungenembolie in der Schwangerschaft [151]

\begin{tabular}{lcc}
\hline Test & $\begin{array}{c}\text { Geschätzte Strahlenbelastung } \\
\text { des Fötus (mSv) }\end{array}$ & $\begin{array}{c}\text { Geschätzte Strahlenbelastung } \\
\text { der weiblichen Brust (mSv) }\end{array}$ \\
\hline Rö-Thorax & $<0,01$ \\
Perfusionsszintigraphie mit Technetium-99m & \\
markiertem Albumin & $0,11-0,20$ \\
Low dose: $40 \mathrm{MBq}$ & $0,20-0,60$ & $0,28-0,50$ \\
High dose:200 MBq & $0,10-0,30$ \\
Ventilationsszintigraphie & $0,24-0,66$ \\
CT-Angiographie & $10-70$ \\
\hline
\end{tabular}

$\mathrm{mSv}=$ Millisievert 
ner Lungenembolie findet sich bei 1-2\% aller CT-ThoraxUntersuchungen mit steigender Tendenz. Am häufigsten sind Patienten mit Tumorerkrankung, aber auch jene mit Herzinsuffizienz oder Vorhofflimmern betroffen [84, 212, 246].

Für Behandlungstrategien der inzidentellen Lungenembolie liegt keine ausreichende Evidenz vor. Analog zum Vorgehen bei inzidenteller Venenthrombose wird in den meisten Fällen eine Antikoagulation in therapeutischer Dosis empfohlen. Das gilt vor allem für Patienten mit zentralen und segmentalen Emboli und solchen bei denen von vornherein ein hohes VTE-Risiko angenommen wird, z. B. bei nachgewiesenem Malignom oder bei chronisch entzündlichen bzw. autoimmunologischen Erkrankungen [141]. Speziell bei den Tumorpatienten gilt dann die Empfehlung für niedermolekulares Heparin über 3 Monate.

\section{Nachsorge der Lungenembolie}

\section{Chronisch-thromboembolische pulmonale Hypertonie (CTEPH)}

Bei der chronisch-thromboembolischen pulmonalen Hypertonie (CTEPH) handelt es sich um eine narbige Obstruktion der Lungenarterien, die von komplexen Wandveränderungen der pulmonalen Widerstandsgefäße begleitet wird [313]. Die CTEPH gilt als eine seltene Langzeitkomplikation nach einer einzigen bzw. nach rezidivierenden Lungenembolien. Die Inzidenz wird aktuell auf 5 Fälle pro 1 Million Personen/Jahr und die Prävalenz auf 3-30 Fälle pro 1 Million Personen/Jahr geschätzt [146].
Angesichts dieser geringen Zahlen wird aktuell ein flächendeckendes "CTEPH-Screening“ asymptomatischer Patienten nach abgelaufener LE nicht empfohlen.

Bei Patienten mit persistierender Dyspnoe nach Lungenembolie sollte eine CTEPH-Diagnostik durchgeführt werden.

Ein CTEPH-Screening sollte bei beschwerdefreien Personen mit einer TVT oder LE in der Anamnese nicht durchgeführt werden.

Jedoch sollte bei allen Patienten, die nach mindestens 3-monatiger therapeutischer Antikoagulation einer Lungenembolie über persistierende Dyspnoe oder reduzierte Belastbarkeit bzw. im weiteren Krankheitsverlauf über neue, für eine LE typische, Beschwerden klagen, eine CTEPH ausgeschlossen werden.

Als erstes Untersuchungsverfahren ist bei Verdacht auf eine CTEPH ein Echokardiogramm durchzuführen. Bei fehlendem konkretem Hinweis auf eine pulmonale Hypertonie ist ergänzend eine Spiroergometrie als sensitives Verfahren zur Einschätzung einer funktionellen Einschränkung der pulmonalen Perfusion zu erwägen. Bei pathologischem oder suspektem Befund in einer dieser Untersuchungen erfolgt als nächster Schritt die bildgebende nuklearmedizinische Diagnostik mittels Ventilations-Perfusionsszintigraphie und anschließend - bei Nachweis von Perfusionsausfällen - die Bestätigung einer präkapillären pulmonalen Hypertonie mittels Rechtsherzkatheter. Radiologische bildgebende Verfahren und insbesondere eine konventionelle Pulmonalisangiographie sind zur definitiven Bestätigung der CTEPH-Diagnose sowie vor pulmonaler Endarteriektomie zur Beurteilung der Operabilität des Patienten erforderlich [313]. 